\title{
EL POBLAMIENTO HUMANO ANTIGUO EN EL VALLE ALTO DEL LOZOYA (MADRID)
}

\section{Ancient human population at the upper Lozoya Valley (Madrid)}

\author{
Belén Márquez, Marina Mosquera, Joaquín Panera, Sergio Bárez, Inmaculada Rus, \\ Juan Gómez, Juan Luis Arsuaga, Alfredo Pérez-González y Enrique Baquedano*
}

Recibido el 10 de diciembre de 2007. Aceptado el 7 de enero de 2008

Resumen. La localización en superficie en el término municipal de Lozoya (Madrid) de un conjunto de piezas de industria lítica achelense permite retrotraer el primer poblamiento de esta zona al Pleistoceno medio, cuando hasta la fecha las evidencias más antiguas en el valle alto del río Lozoya se remontaban a inicios del Pleistoceno superior.

El nuevo hallazgo sitúa al río Lozoya en relación cultural con el poblamiento humano antiguo de los ríos Jarama y Manzanares.

Palabras clave: Paleolítico inferior. Pleistoceno medio. Achelense, Alto Valle del Lozoya. Madrid, España.

Abstract. The discovery of a surface sample of Acheulean lithic industry at the Lozoya district (Madrid) takes back to the Middle Pleistocene the first human peopling of this area. Until now the most ancient evidences at the Upper Lozoya Valley went back to the initial Upper Pleistocene.

The new findings place the Lozoya River in cultural relation with the ancient human population of Jarama and Manzanares Rivers.

Key Words: Lower Palaeolithic. Middle Pleistocene. Acheulean, Upper Lozoya Valley. Madrid, Spain.

\section{INTRODUCCIÓN}

Durante la campaña de excavaciones de 2005 en los yacimientos del Valle Alto del Lozoya, se descubrió, en terrenos pertenecientes al término municipal de Lozoya del Valle, un conjunto de industria lítica atribuible al tecnocomplejo achelense.

Hasta la fecha las evidencias más antiguas conocidas de ocupaciones humanas en el Valle Alto del Lozoya habían sido las proporcionadas por los yacimientos del Calvero de la Hi- guera en el municipio de Pinilla del Valle. Este paraje contiene un karst de dolomías de edad cretácica, con al menos tres cavidades que en su momento fueron ocupadas por homínidos (Homo neanderthalensis) y hienas (Crocuta crocuta).

Las investigaciones actuales emprendidas por nuestro equipo a partir de 2002 han permitido situar la fecha para la presencia humana en la Cueva del Camino a inicios del Pleistoceno superior (datación por TL de $90.961 \pm 7.881$ BP1), descartando una edad meso-pleistocena, previamente postulada, para di-

(*) Belén Márquez y Enrique Baquedano: Museo Arqueológico de la Comunidad de Madrid. Plaza de las Bernardas, s/n. 28801-Alcalá de Henares (Madrid).E-mail: belen.marquez@madrid.org; enrique.baquedano@madrid.org

MARINA MosQueRA:Universitat Rovira i Virgili, Ȧrea de Prehistòria, Plaça Imperial Tàrraco, s/n 43005-Tarragona. E-mail: marina.mosquera@urv.net JoAquín PANERA: E-mail: joaquin.panera@gmail.com

SERGIo BÁREZ: E-mail: paleosergio@yahoo.es

InMACULADA RUS: Dirección General de Patrimonio Histórico. Consejería de Cultura y Turismo de la Comunidad de Madrid. C/Arenal, 18, 28013Madrid. E-mail: inmaculada.rus@madrid.org

JUAN GómEz: E-mail: juangom@ole.com

JuAN LUIS ARSUAGA: Centro de Investigación UCM-ISCIII sobre Evolución y Comportamiento Humanos C/Sinesio Delgado, 4, 28029-Madrid. E-mail. jlarsuaga@isciii.es

Alfredo Pérez-GonzÁlez: Centro Nacional de Investigación sobre Evolución Humana (CEMEN). Avda. La Paz, 28, entreplanta. 09004 Burgos. E-mail: alfredo.perez@cenieh.es

(1) Todas las dataciones por TL aqui citadas proceden del Laboratorio de Datación y Radioquímica de la Universidad Autónoma de Madrid. 
chos restos (Alférez et al., 1982). Asimismo, hemos llegado a determinar (Arsuaga et al., e.p.) que el homínido que aparece en dichas cavidades pertenece a la especie Homo neanderthalensis y no a los "anteneandertales", como proponen sus descubridores, tratándose, igualmente, esta cavidad de un cubil de hienas (Díez, 1993), y no de un lugar frecuentado por homínidos. Este yacimiento, asimismo, ha proporcionado una asociación faunistica muy amplia característica de este periodo.

Por otra parte, la Cueva de la Buena Pinta, descubierta en 2003, que presenta niveles holocenos en el tramo superior de la secuencia, fue igualmente utilizada como cubil de hienas, aunque también muestra evidencias de episodios breves de ocupación humana. El nivel 3 de este yacimiento ha ofrecido una datación por TL de 63.451+-5.509 BP.

Por ultimo, el Abrigo de Navalmaíllo, descubierto en 2002, contiene hasta el momento varios niveles con industria musteriense y evidencias de actividades relacionadas con la vida cotidiana de Homo neanderthalensis. Las dataciones

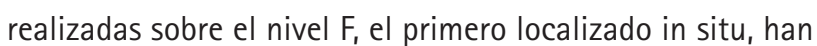
arrojado una edad de $71.685 \pm 5.082$ y de $77.230 \pm 6.016$ BP (Arsuaga et al., e.p.), que se corresponden con el final del estadio isotópico 5.

En el marco de este proyecto se ha hallado el conjunto de útiles líticos achelenses objeto del presente trabajo.

\section{EL ACHELENSE EN LA CUENCA DE MADRID}

En la cuenca de Madrid, el Achelense se ha identificado con los hallazgos de los cursos medio y bajo de los ríos Manzanares y Jarama, donde sus grandes paleo-llanuras aluviales favorecieron la abundancia de recursos naturales necesarios para la vida de los homínidos. Sin embargo, para comprender los modelos de ocupación de la cuenca de Madrid por parte de los homínidos durante el Pleistoceno medio, es necesario prestar atención a los cursos altos de los ríos Manzanares y Jarama y de sus tributarios, que debieron formar biotopos complementarios que, a su vez, jugaron un importante papel, bien por la estacionalidad de los recursos, bien por constituir zonas de tránsito entre las dos submesetas.

En este sentido, el curso alto del Jarama, y en especial sus afluentes por la margen derecha, puede proporcionar una relevante información a tenor de los hallazgos registrados aguas arriba de Talamanca del Jarama, a lo largo de los arroyos San Vicente y del río Lozoya.

La cuenca de Madrid cuenta con un dilatado periodo de investigación del Paleolítico que se remonta a 1863, momento en el que se reconoce internacionalmente la identificación que el ingeniero Casiano de Prado hizo posible de la industria lítica del madrileño cerro de San Isidro. Además, este pionero de la Prehistoria en España en su excepcional "Descripción física y geográfica de la Provincia de Madrid" (Prado, 1864). señaló el potencial arqueológico de la cuenca alta del Jarama.
Desde entonces, tan sólo la Cueva de El Reguerillo ( $\mathrm{Pa}$ tones, Madrid), en la Sierra madrileña, centró la atención de algunos investigadores (Breuil, 1920; Loriana, 1942; Maura, 1952). Hay que esperar a que en los años ochenta un equipo de paleontólogos excave la cavidad referida en Pinilla del Valle donde se descubren dos molares que atribuyeron a homínidos anteneandertales (Alférez et al., 1982).

Las primeras referencias al achelense en la zona se producen en la tesis de licenciatura de P. Cabra, donde cita un depósito pleistoceno de origen fluvio-lacustre, hallado a los pies de la localidad de Redueña, con industria lítica achelense y restos paleontológicos (Cabra, 1981; Cabra et al., 1983). Con posterioridad I. Rus realiza prospecciones intensivas en el área, que permiten obtener una muestra más amplia compuesta por bifaces, hendedores, núcleos y un pequeño repertorio de útiles sobre lascas que atribuyó al Achelense medio (Rus, 1987).

En las prospecciones realizadas para la elaboración de la Carta Arqueológica de la Comunidad de Madrid se producen nuevos hallazgos achelenses (fig. 1). Los conjuntos más amplios e interesantes se localizan en depósitos asociados al arroyo de San Vicente y su tributario arroyo de Las Huertas en los términos municipales de Redueña y Torrelaguna. Se trata de muestras de superficie recogidas sin contextos geomorfológicos o estratigráficos, hallazgos que incrementa M. A. Valero en el marco de su tesis de licenciatura (Valero, 1995).

El depósito fluvio-lacustre de Redueña fue objeto de investigación a fines de 2003, sin que fuera posible establecer relación entre este depósito y la industria lítica hallada en superficie (Rus et al., 2007).

En el Manzanares, se han documentado industrias achelenses en la terraza de $+25-30$ m (cota a muro), en los niveles inferiores de San Isidro y de Transfesa, mientras que los niveles de $+20 m$ y $+12-15 m$, último tramo del Pleistoceno medio y primer tramo del Pleistoceno superior respectivamente, podrian contener conjuntos atribuibles al Achelense final/Musteriense. En el Jarama, las unidades litoestratigráficas de Arganda I y II de su terraza compleja, que estarían representando la segunda mitad del Pleistoceno medio, son las que contienen industria achelense.

La unidad Arganda I, a partir de los micromamíferos del yacimiento Áridos 1 que se ubica en esta unidad, se sitúa entre Cúllar Baza y los niveles TD 10 y G II de Atapuerca (Sesé y Sevilla, 1996; Santonja, Pérez-González, Villa, et al., 2001), lo que según las dataciones propuestas para Atapuerca (Pérez-González et al., 2001) la fecharía en los estadios isotópicos 9 u 11. En la terraza de $+30 \mathrm{~m}$ del Manzanares los restos de Praedama sp. descritos en Transfesa, junto con la morfología poco evolucionada del Elephas (P) antiquus, señalan que no está lejos del límite Bihariense-Oldenburgiense (Sesé y Soto, 2000), es decir, en torno a los estadios isotópicos 11-13 (Santonja, et al., 2001). En el Jarama la T + 


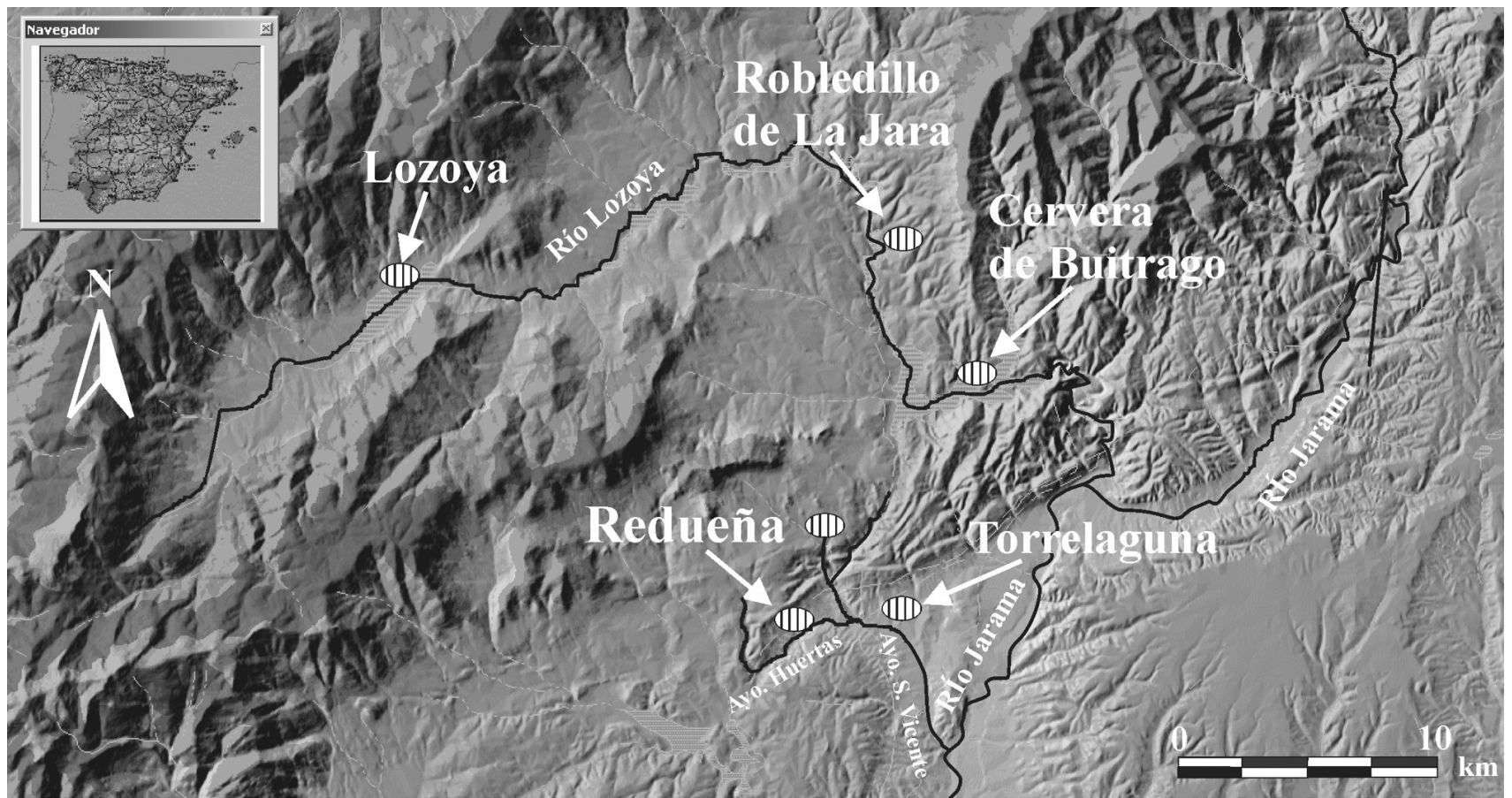

- Figura 1. Situación de los principales enclaves con industria achelense en la sierra norte de la Comunidad de Madrid.

40 m no está aparentemente afectada por el fenómeno de subsidencia sinsedimentaria, por lo que cabría suponer una equivalencia entre la T $+30 \mathrm{~m}$ con la unidad Arganda I (Pérez-González y Uribelarrea 2002), y por tanto una correspondencia cronológica entre Arganda I y la $\mathrm{T}+30 \mathrm{~m}$ del Manzanares. De este modo, los datos parecen apuntar a que los vestigios achelenses en la cuenca de Madrid se pueden remontar al estadio isotópico 11 (427-364 ka).

Como ya se ha señalado (Rus et al., 2007), los escasos datos disponibles de industria achelense en la cuenca alta del Jarama sugieren una serie de ocupaciones en torno a los dos afluentes principales del río Jarama por su margen derecha: el río Lozoya y el arroyo de San Vicente.

En torno al río Lozoya se han hallado algunos bifaces aislados o asociados a algún núcleo, y en la cabecera del arroyo de San Vicente se han localizado sendos bifaces, uno junto a un hendedor ${ }^{2}$. En el tramo medio de este arroyo se han documentado cuatro concentraciones de industria achelense, compuesta por bifaces y hendedores (García Valero, 1995), y en Redueña -en el entorno del arroyo de las Huertas, tributario del anterior-, una muestra compuesta por hendedores, bifaces y lascas retocadas.

De todos estos hallazgos, sólo se ha publicado la muestra de Redueña (Rus et al., 2007), en la que priman los hendedores y bifaces frente a núcleos y lascas, la mayoría de cuarcita. Las lascas de grandes dimensiones han sido los soportes preferidos para desarrollar estos útiles, y debido a la ausencia en el área del mismo tipo de cuarcitas y de núcleos y nódulos de los que se pudieran haber extraído, se ha sugerido que se llevaron configuradas desde las terrazas del Jarama a unos $5,5 \mathrm{~km}$.

\section{CONTEXTO GEOLÓGICO DEL NUEVO YACIMIENTO ACHELENSE}

La cabecera del valle del río Lozoya se encuentra en el sector central del Sistema Central español drenando la vertiente sur del Guadarrama (Peñalara, 2.428 m), la norte de Cuerda Larga y los Altos del Hontanar, en la Sierra de la Morcuera $(1.834 \mathrm{~m})$.

El Sistema Central Español es un "pop-up" cenozoico con orientaciones E-W a NE-SW (de Vicente et al., 2007), que, en su sector central presenta una depresión tectónica o popdown, con sedimentos preservados del Cretácico y Terciario, que conforma el valle alto del río Lozoya.

Los materiales más antiguos de edades precámbricas y paleozoicas están representados por esquistos, pizarras y cuarcitas que han sufrido un intenso metamorfismo y deformación durante el Varisco. Sin embargo, las rocas con mayor extensión areal en el entorno del embalse de Pinilla son los ortoneises y los granitos. Las rocas filonianas más comunes son los cuarzos, lamprófidos y pórfidos (Bellido et al., 1988).

Sobre estos materiales se apoyan depósitos alpinos siliciclásticos y carbonatados marinos del Cretácico superior y a su techo sedimentos detríticos gruesos continentales de edad ter-

(2) Carta Arqueológica de la Comunidad de Madrid. Documentación depositada en la Dirección General de Patrimonio Histórico. Consejería de Cultura y Deportes de la Comunidad de Madrid (J.F. Pastor Muñoz 1993 y 1994). 

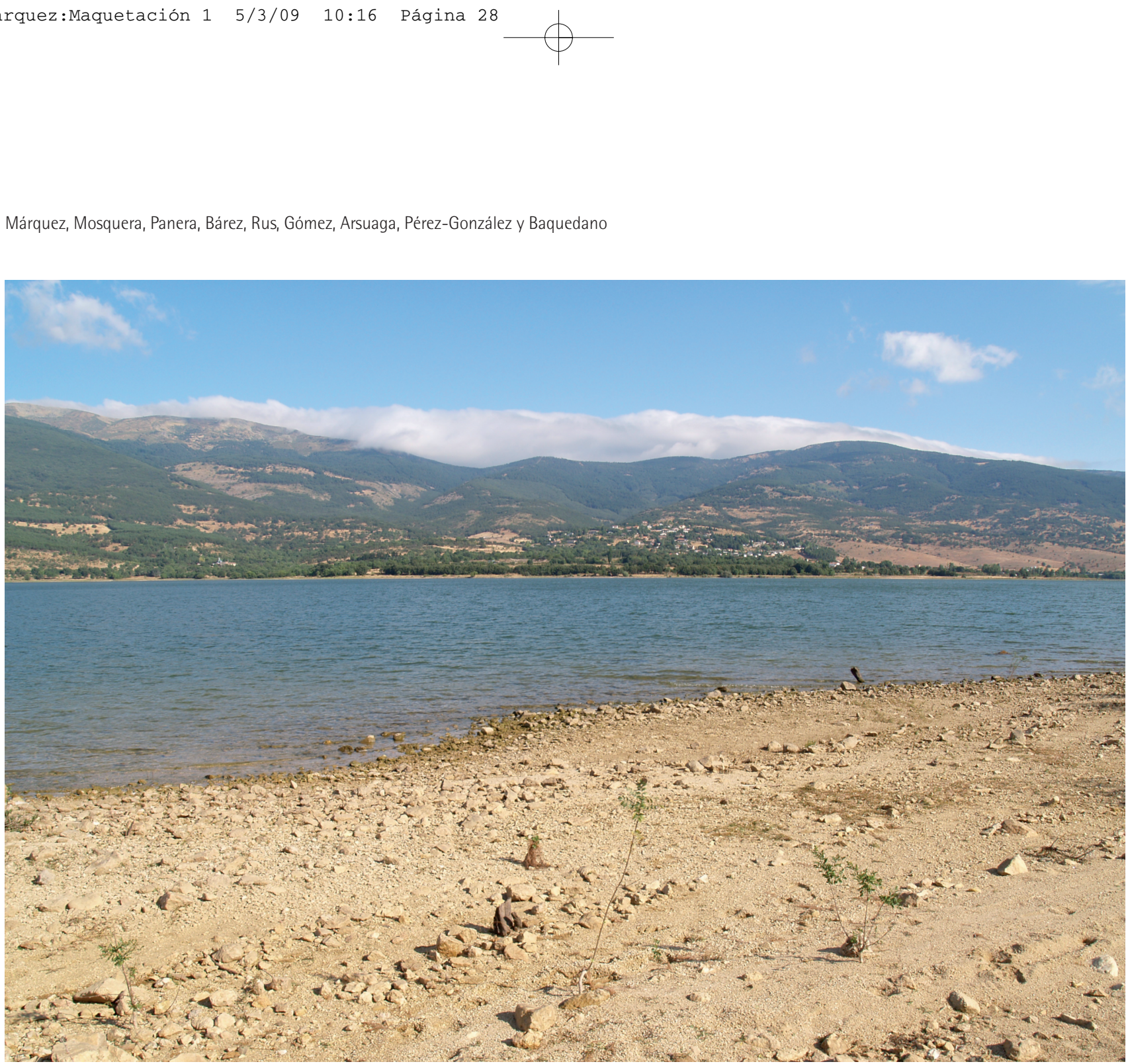

- Figura 2. Vista del yacimiento. Puede apreciarse el municipio de Lozoya al otro lado del embalse.

ciaria. El Cuaternario alcanza su mejor expresión a lo largo del río Lozoya y está constituido por terrazas fluviales, abanicos y glacis con cobertera. En los relieves dominantes circundantes se han cartografiado y descrito formas glaciares y morrenas. Igualmente, hay otros elementos periglaciares y niveles, como suelos estructurados, procesos solifluidales, nichos nivales, etc (Bellido et al., 1988; Arenas Martín et al., 1986-87).

Las formas y depósitos cársticos mejor conocidos desarrollados a favor de las rocas carbonatadas cretácicas son los del Calvero de la Higuera en el término de Pinilla del VaIle (Arsuaga et al., e.p.).

Al NE del Calvero de la Higuera y asociado al río Lozoya, en su margen derecha o a depósitos de origen lateral, se sitúa el yacimiento achelense estudiado en este trabajo. Se trata de un depósito de muy escasa potencia, probablemente desmantelado por acciones erosivas posteriores a su sedimentación, que descansa sobre un Cretácico asociado cuarzo-calizo y arenoso, con buzamiento hacia el norte (fig. 2)

Estos materiales aluvionares siliciclásticos se sitúan morfológicamente a unos $+10 \mathrm{~m}$ sobre el cauce del río Lozoya, por lo que podría tratarse de una terraza fluvial, pero también de restos conservados de un abanico aluvial, ya que un pequeño barranco desagua inmediatamente al norte del yacimiento (fig. 3).

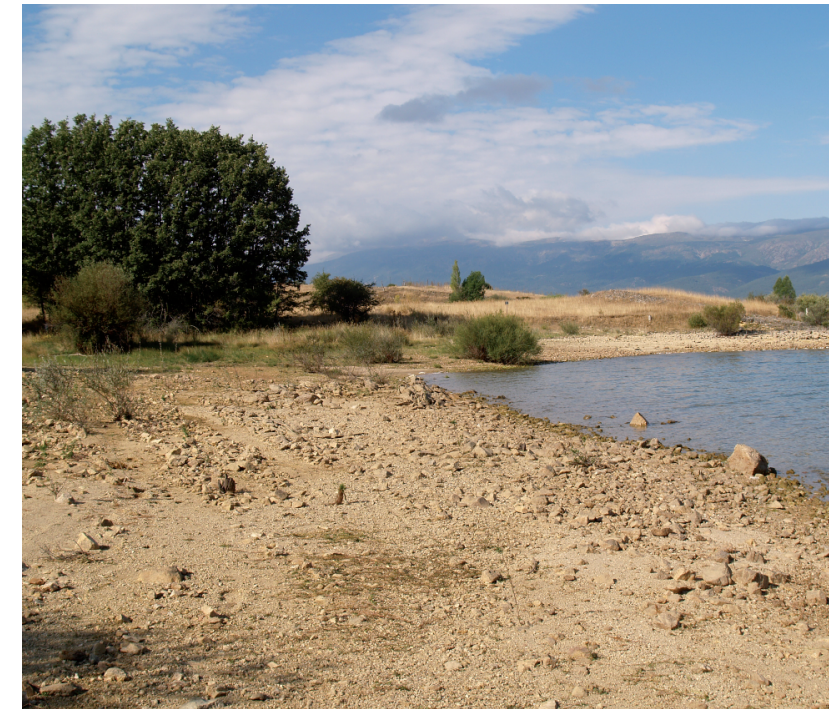

A Figura 3. Vista del yacimiento. Al fondo, se divisa la cumbre de Peñalara.

Es de esperar que prospecciones sistemáticas geológicas, geomorfológicos y arqueológicas en un futuro próximo, permitan aclarar el contexto morfoestratigráfico de estas industrias y por ende precisar su cronología de probable edad en el Pleistoceno medio avanzado. 


\section{EL CONJUNTO LÍTICO}

El conjunto estudiado está formado por 11 piezas: siete grandes instrumentos, tres lascas de mediano formato y un núcleo. La muestra está mayoritariamente elaborada sobre diferentes variedades de cuarcita, a excepción de dos lascas que están realizadas en silex y otra roca silícea criptocristalina. El conjunto presenta distintos grados de erosión. En ese sentido se pueden diferenciar tres grandes grupos entre las que muestran un grado de erosión mayor (fig. 4: 3; fig 5; fig. 8:1), medio (fig. $4: 1$ y 2 ; fig. 6 y fig. 7) y menor, la cual se corresponde con el único núcleo de la muestra (fig. 8: 2).

Las tres lascas han sido elaboradas en tres tipos de materiales: silex, cuarcita y otra roca silícea criptocristalina. Son

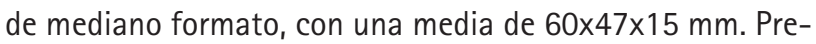
sentan plataformas talonares diversas: cortical, unifacetada y bifacetada. Las caras dorsales son predominantemente no corticales, mostrando un máximo de 2-3 levantamientos.

El único núcleo de la muestra es de cuarcita. Se trata de un unifacial unipolar masivo sobre canto, en el que un pequeño segmento de la pieza ha sido tratado bifacialmente de cara a preparar la plataforma de percusión, descortezando la superficie. En la plataforma de explotación se alternan las extracciones de mediano y pequeño tamaño, siendo todas ellas en general menores de $40 \mathrm{~mm}$. En la reserva cortical se observa un ángulo del canto con marcas de percusión que permiten pensar que la pieza ha podido ser utilizada en algún momento como percutor.

En cuanto a los grandes instrumentos, dos de ellos han sido elaborados sobre canto, mientras que el resto ha sido tallado sobre grandes lascas. Respecto a estos últimos, todos ellos presentan el eje técnico de la lasca soporte desviado respecto al morfológico del bifaz. Se trata de un hendedor y seis bifaces, dos apuntados y tres ovalados. El tamaño medio de este conjunto es de $134 \times 81 \times 45 \mathrm{~mm}$, con una longitud máxima y mínima de $164 \mathrm{~mm}$ y $102 \mathrm{~mm}$, respectivamente.

El hendedor se corresponde con el tipo 0 de Tixier, mostrando los dos laterales configurados y el filo distal natural. Técnicamente este hendedor y dos de los bifaces son unifaciales. En general, todos los instrumentos de grandes dimensiones presentan una configuración notablemente intensa por una sola cara, elaborada a partir de una única generación de levantamientos muy profundos o totales, con objeto de configurar la superficie de esa cara. En particular, una de las piezas presenta un tratamiento bifacial más intenso, aunque mantiene una sola generación de levantamientos. Destacamos el caso de un bifaz en el que puede rastrearse el orden de las tres generaciones de levantamientos que se realizaron para configurar su filo distal denticulado convexo.

Pocos son los datos que nos informan sobre la correspondencia tecnológica de este conjunto, ya que el método de explotación presente en el núcleo no es diagnóstico de ninguna atribución cultural o cronológica, No obstante, cabe

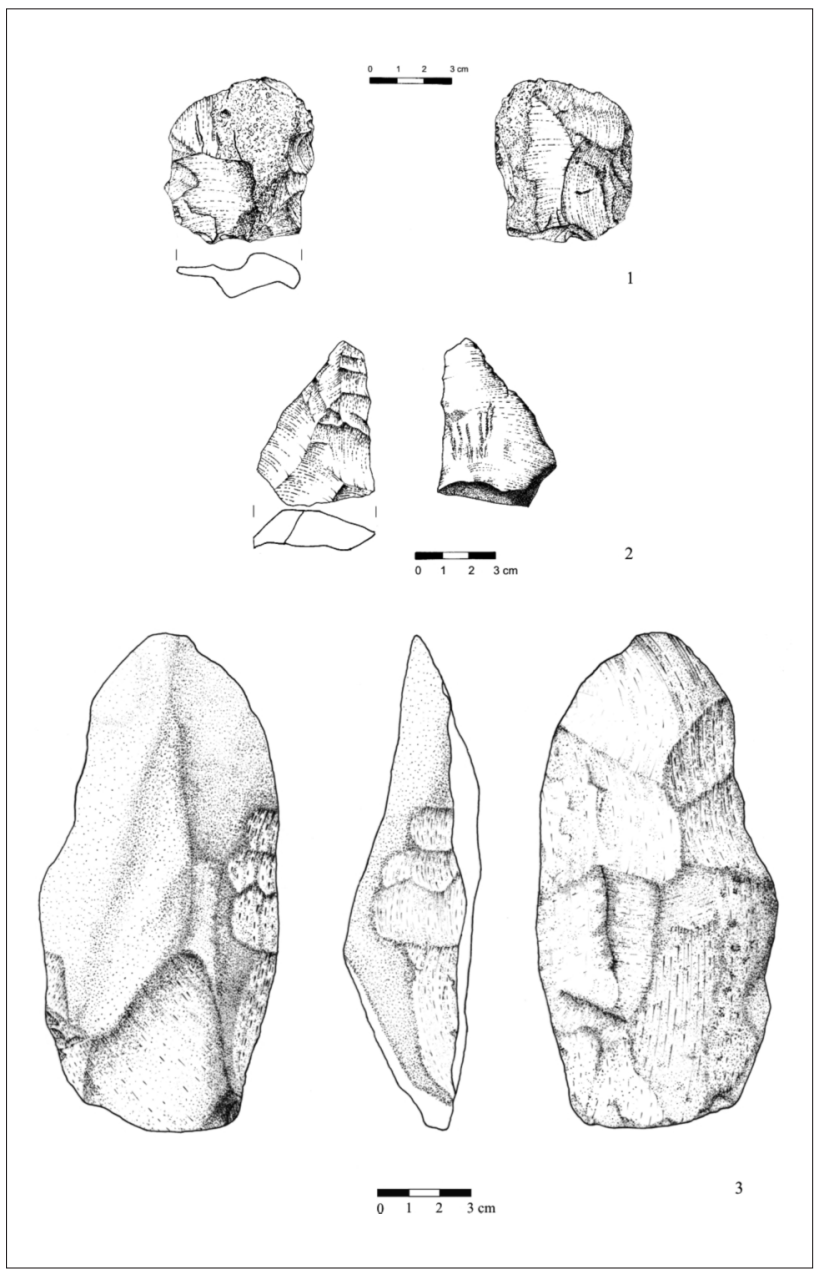

- Figura 4: 1. Lasca simple de roca silícea criptocristalina; 2. Lasca simple de sílex; 3. Bifaz de cuarcita.

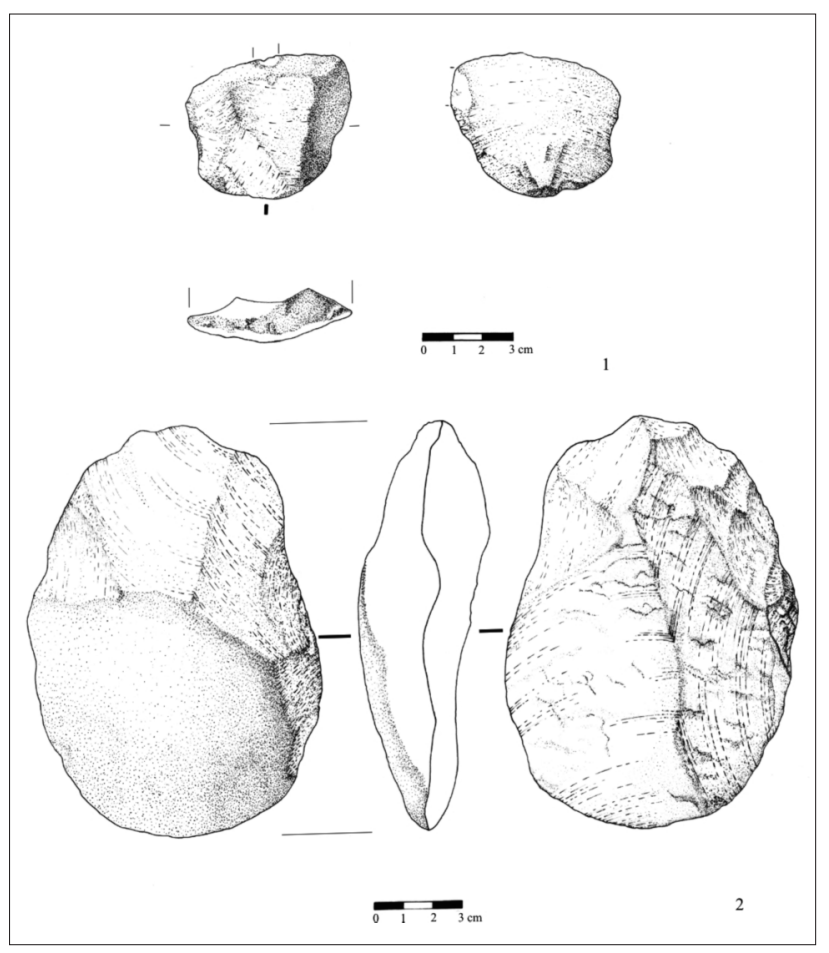

A Figura 5: 1. Lasca simple de cuarcita; 2: Bifaz de cuarcita. 


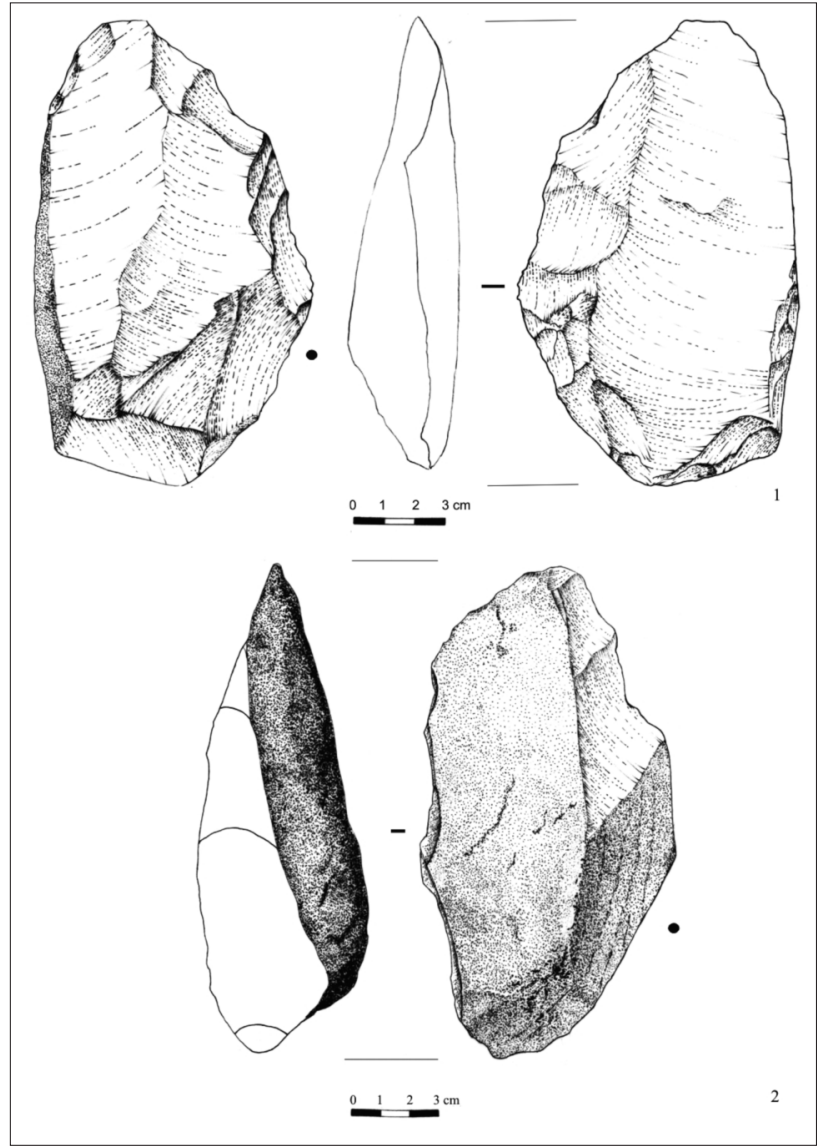

A Figura 6: 1. Bifaz parcial sobre lasca de cuarcita; 2. Hendedor tipo 0 de cuarcita.

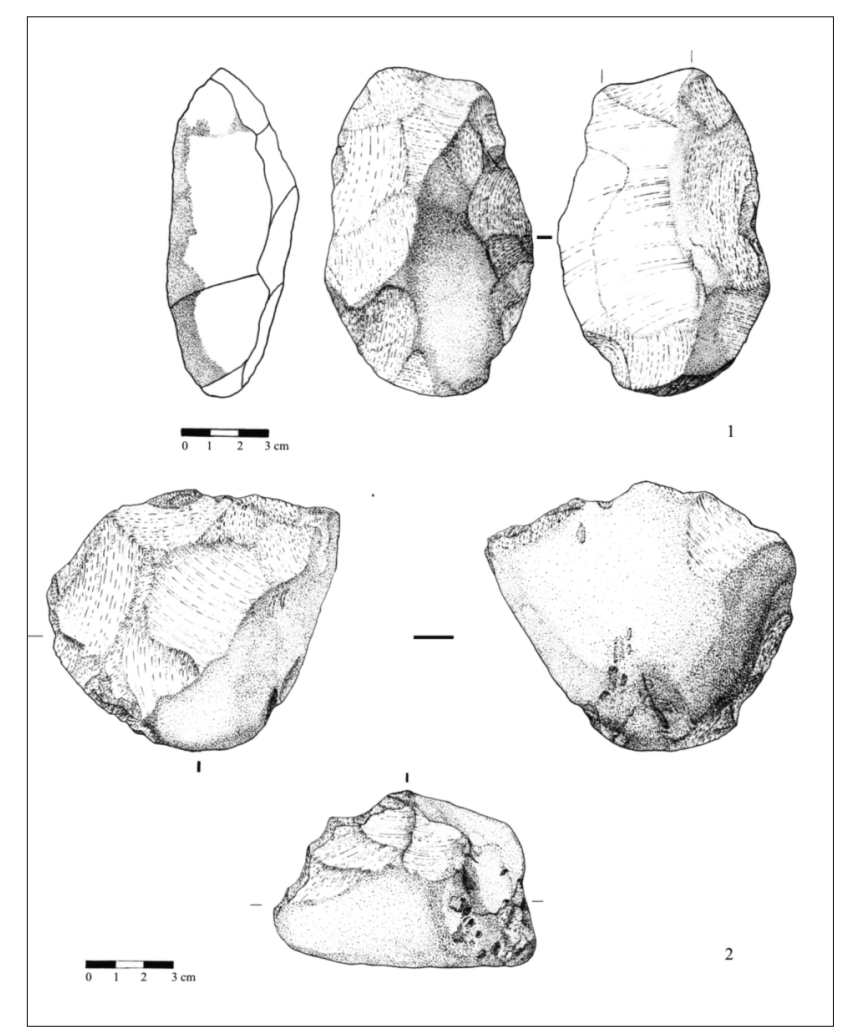

A Figura 8: 1. Bifaz de cuarcita; 2. Núcleo de cuarcita.

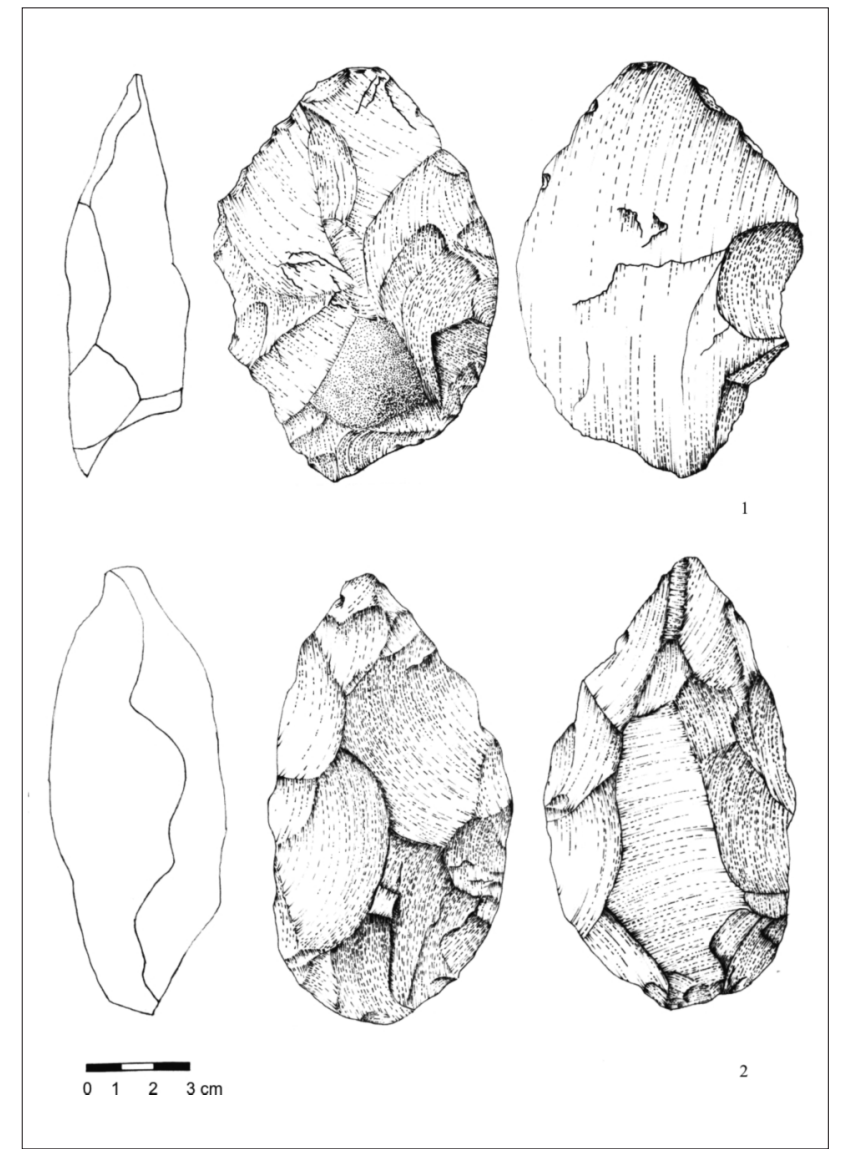

A Figura 7: 1 y 2. Bifaces de cuarcita.

señalar que la presencia de grandes instrumentos sobre lasca apunta a un tecno-complejo achelense. De otra parte, la desviación del eje técnico respecto al morfológico de este tipo en piezas -observable en otros conjuntos mesopleistocenos, como Torralba y Ambrona- sugiere que no se trataría de un achelense tardío en exceso.

\section{CONCLUSIÓN}

El nuevo hallazgo de industria lítica achelense en el valle alto del río Lozoya viene a confirmar que dicha zona tuvo un poblamiento anterior al Pleistoceno superior del Calvero de la Higuera. Al tiempo incorporaría la sierra madrileña al listado de estaciones inferopaleolíticas de la cuenca madrileña.

Con este análisis previo hemos querido, junto al ofrecimiento del sentido tributo al profesor Ripoll, ofrecer más datos para construir el rompecabezas del poblamiento homínido en el interior peninsular. Las recientes fechas obtenidas en algunos yacimientos con industrias musterienses (Ambrona, Cuesta de la Bajada, Gran Dolina, etc.; Santonja y Pérez-González, 2006), abren una apasionante línea de investigación sobre el proceso evolutivo, tanto físico como TECNO-cultural, de Homo heidelbergensis a Homo neanderthalensis. 


\section{AGRADECIMIENTOS}

Pilar Sala y Natalia Suárez han realizado los dibujos de industria lítica.

\section{BIBLIOGRAFÍA}

Alférez, F., G. Molero, Maldonado, E., Bustos, V., Brea, P., y Buitrago, A. M.: Descubrimiento del primer yacimiento cuaternario (Riss-Würm) de vertebrados con restos humanos en la provincia de Madrid (Pinilla del Valle). Coloquios de Paleontología, 37 (1982), pp.15-32.

Arenas Martín, R.; Fuster, J. M.; Martínez-Salanova, J.; Del Olmo, A. y VILASECA, C.: Hoja Geológica a Escala 1:50.000, de Segovia (483). (1986-87). IGME.

Arsuaga, J. L., Baquedano, E. y Pérez-González, A.: Neanderthal and carnivore occupations in Pinilla del Valle Sites (Community of Madrid, Spain) (e.p.). Proceedings of the XV UISPP Congress. Lisboa 2006.

Bellido, F.; Escudero, J.; Klein, E.; Del Olmo, A.: Hoja Geológica a Escala 1:50.000, de Buitrago del Lozoya (484). (1988). IGME.

BreUIL, H.: Miscellanea d'art rupestre - Cueva del Reguerillo près Torrelaguna (Madrid). Boletín de la Real Sociedad Española de Historia Natural, XX (1920): 376.

CABRA, P.: Estudio geomorfológico y de las formaciones superficiales entre la Sierra de La Cabrera y la superficie de Mesones (1981), Tesis de Licenciatura. Universidad Complutense de Madrid.

CABRA, P; GoY, J. L.; HoYos, M.; ZAZO, C.: Estudio geomorfológico del Cuaternario y de las formaciones superficiales del sector meridional de la Sierra Cabrera. Tecniterrae, 51 (1983): 32-42.

Díez Fernández-Lomana, C.: Zooarqueología de Atapuerca (Burgos) e implicaciones paleoeconómicas del estudio tafonómico de yacimientos del Pleistoceno medio. (1993) Tesis Doctoral, Universidad Complutense de Madrid.

Garcia Valero, M. A.: El Poblamiento paleolítico en los valles altos del Jarama y del Sorbe (inédita). Trabajo de Investigación, 1995. Departamento de Prehistoria y Etnología. Facultad de Geografía e Historia. Universidad Complutense de Madrid.

MARQUÉS DE LORIANA.: Grabados auriñacienses en una cueva de la provincia de Madrid. Archivo Español de Arqueologia, 46 (1942): 76-78.

MaURA, M.: Los dibujos rupestres de la Cueva del Reguerillo (Torrelagu- na). Provincia de Madrid. II Congreso Nacional de Arqueología (Madrid, 1951) (1952): 73-74. Zaragoza.

Pérez González, A.; Parés, J. M.; Carbonell, E.; Aleixandre, T.; Ortega, A. I.; Benito, A. y Martín Merino, M. E.: Geologie de la Sierra de Atapuerca et stratigraphie des remplissages karstiques de Galería et Dolina. L'Anthropologie, 95 (1995). Paris.

Pérez-GonzÁlez, A. y Uribelarrea Del VAl, D.: Geología del Cuaternario de los valles fluviales del Jarama y Manzanares en las proximidades de Madrid. En Bifaces y Elefantes. La investigación del Paleolítico inferior en Madrid. Zona Arqueológica, 1 (2002): 302-317. Museo Arqueológico Regional de la Comunidad de Madrid. Alcalá de Henares.

PrAdo, C. DE.: Descripción física y geológica de la provincia de Madrid. Madrid. (1864). Junta General de Estadistica. Madrid (Reedición: (1975). Colegio de Ingenieros de Caminos Canales y Puertos.

Rus, I. "El Paleolítico". 130 Años de Arqueología Madrileña. (1987): 2043. Consejería de Cultura y Deportes de la Comunidad Autónoma de Madrid.

Rus, I; Panera Gallego, J.; Rubio Jara, S., Pérez-González, A.; Bárez, S.: La Cuenca Alta del río Jarama: una aproximación geoarqueológica al depósito Pleistoceno de Redueña. Zona arqueológica, 7 (1) (2006): 195-212. Alcalá de Henares, Madrid.

SAntonja, M. y PéRez-GonzÁlez, A.: La industria lítica del miembro estratigráfico medio de Ambrona (Soria, España) en el contexto del Paleolitico antiguo de la Península lbérica, Zephyrus, LIX (2006):7-20.

Santonja, M. y Pérez-González, A.; Villa, P; Soto, E. y Sesé, C.: Elephants in the archaelogical sites of Aridos (Jarama valley, Madrid, Spain). The World of Elephants. Proceedings of the Io International Congress (2001): 602-606. Roma.

SESÉ, C. Y SEVILLA, P.: Los micromamíferos del Cuaternario peninsular español. Cronoestratigrafía e implicaciones biogeográficas. Revista Española de Paleontología, $n^{\circ}$ extraordinario (1996): 278-287.

SESÉ, C. y SoTo, E.: Vertebrados del Pleistoceno del Jarama y Manzanares en En Bifaces y Elefantes. La investigación del Paleolítico inferior en Madrid. Zona Arqueológica, 1 (2000): 318-337. Museo Arqueológico Regional de la Comunidad de Madrid. Alcalá de Henares.

Vicente, G. De.; Vegas, R.; Muñoz Martin, A.: Silva. P. G.; Andriessen, P.; Cloetingh, S.; González Casado, J. M.; Van Wees, J. D.; Álvarez, J.; CARBó, A. y OLAIZ, A.: Cenozoic tic-skinned deformation and topography evolution of the Spanish Central System, Global and Planetary Change, 58 (2007): 335-381. 